\title{
Galaxies and cladistics
}

\author{
Didier Fraix-Burnet
}

September 15, 2009

Laboratoire d'Astrophysique de Grenoble, UMR5571 CNRS/Université Joseph Fourier

didier.fraix-burnet@obs.ujf-grenoble.fr

\author{
Original text published in \\ Evolutionary Biology. Concept, Modelization and Application, 2009, Pontarotti, P. (Ed.), Springer, pp. 363 - 378 \\ DOI 10.1007/978-3-642-00952-5
}

\begin{abstract}
The Hubble tuning fork diagram, based on morphology and established in the 1930s, has always been the preferred scheme for classification of galaxies. However, the current large amount of multiwavelength data, most often spectra, for objects up to very high distances, asks for more sophisticated statistical approaches. Interpreting formation and evolution of galaxies as a 'transmission with modification' process, we have shown that the concepts and tools of phylogenetic systematics can be heuristically transposed to the case of galaxies. This approach that we call "astrocladistics", has successfully been applied on several samples. Many difficulties still remain, some of them being specific to the nature of both galaxies and their diversification processes, some others being classical in cladistics, like the pertinence of the descriptors in conveying any useful evolutionary information.
\end{abstract}

\section{Introduction}

Galaxies have been discovered quite recently with respect to the History of Humanity, since it was in 1922 by Edwin Hubble. Our own galaxy, called the Milky Way, was rather well known at this epoch. Hubble found that there are indeed many other similar objects situated at very far distances from us, much farther away than the dimension of the Milky Way. Galaxies are now known to be fundamental entities of the Universe and can be defined as self-gravitating ensemble of stars, gas and/or « dust $\gg$ (dust being mainly grains for astronomers).

Typical big galaxies like our own are about 100000 light-years across. Shapes are basically those defined by Hubble, that is spiral, elliptical and irregular. This reflects the orbits of the stars that can be distributed in a disk (spirals), in a regular three dimension structure (ellipticals), or in a more disturbed fashion (irregulars). In spiral galaxies, density waves create spiral arms, sometimes with a bar-like structure around the centre of the galaxy. These density waves are not necessarily long-lasting, but they tend to concentrate gas and dust so that star formation is mostly associated with them.

Self-gravitating objects have probably appeared early in the Universe (which age is now established to be 13.7 $10^{9}$ years), in association with the very slightly inhomogeneous distribution of gravitational matter. The structure of the Universe is shaped by the dark energy ( $70 \%$ of the Universe total energy), that causes the expansion, and the gravitational field that is fashioned essentially by the dark matter (26\%). Galaxies are made up of baryonic matter which represents only $4 \%$ of the composition of the Universe. The primeval inhomogeneities of the gravitational potential are observed at the recombination epoch (time when the Universe became transparent at an age of about 400000 years) thanks to their thermal radiation that the expansion of the Universe has now "cooled" down to $3 \mathrm{~K}$ (e.g. Hinshaw et al. 2009)). These early overdensities have grown up by condensation to form bigger and bigger structures called halos and filaments. The baryonic matter is gravitationally affected by the evolution of the dark matter distribution, and collapses of small scale entities created the first light emitting objects (first stars at about 400 millions years) whose distribution can be observed now in the infrared (Kashlinsky et al. 2005). Currently, most distant galaxies can be seen at redshifts about 6 or 7 , equivalent to distances of about 12 Gyr or $90 \%$ of the age of the Universe in lookback time. Since the Universe is in expansion, it was denser at these earlier epochs, so that interactions and collisions between self-gravitating opjects were frequent. This certainly explains why galaxies 
observed at large distances seem rather disturbed and do not fit the Hubble morphological classification (van den Bergh 1998).

There are typically about $10^{8}-10^{12}$ stars in a galaxy. Once formed from the collapse of a cloud of gas and dust, stars evolve by themselves depending on their initial mass and chemical composition. The environment has very little effect on them, they essentially never collide, die, nor disappear. Only the most massive ones explode as supernovae, injecting into the interstellar medium some of their gas that is enriched in heavy atomic elements ("metallic", i.e. heavier than Beryllium and Lithium) and leaving a dense remnant that can be a neutron star or a small black hole.

Galaxies generally have a lot of gas and dust which properties evolve because of stellar radiation and density perturbations. The chemistry of atoms, molecules and grains is local and very complex, but the orbital motions rather rapidly homogenize somehow the chemical properties within a galaxy. In particular, the collapse of molecular clouds that leads to the formation of stars generally happens more or less simultaneously over a significant fraction of the volume of the galaxy. This phenomenon relates global characteristics of galaxies with small scales processes, but it also smears out traces of past localized events.

The properties of the three fundamental constituents (stars, gas and dust) fully describe a given galaxy, provided we have access to them at all places in the three dimensions. This implies that the full description of a galaxy is already complex. Moreover, any gravitational perturbation, either caused by external passing-by galaxies or by internal interactions, modifies the shape of the galaxy, and possibly properties of gas and dust. Even internal events, like the explosion of many supernovae at nearly the same time or density waves, may have a strong influence. Since there are so many components in a single galaxy, since their mutual interactions through radiation, shock waves, gravitational perturbations, chemical and physical processes and so on, are essentially non-linear and chaotic, the evolution of a galaxy truly belongs to the complex sciences.

Nowadays, observations are becoming very detailed and numerous. Systematic surveys feed databases comprising millions of galaxies for which we have images and spectra. Big telescopes and very sensitive detectors allow us to observe objects from the distant past (galaxies at high redshifts). This is somewhat reminiscent of paleontology, and like evolutionary biologists, astronomers want to understand the relationships between distant and nearby galaxies, like our own. Strangely enough, the Hubble classification, born from the Hubble diagram, is still frequently used as a support to describe galaxy evolution, even though it ignores all observables except morphology (i.e. Hernandez and Cervantes-Sodi 2006, Cecil and Rose 2007). Indeed, the Hubble classification is very successful not only because it is simple but also because the global shape of a galaxy grossly summarizes several physical properties. In particular, star formation is more efficient in disks and it seems that spiral galaxies have also more gas than ellipticals. Obviously, the distribution of the stellar orbits is related to the history of a galaxy. Numerical simulations are easier to make with stars and gravitation only, the gas and dust components requiring more complicated equations and much more computer power. Thus, many simulations have shown precisely how the structures of galaxies transform themselves during interactions or merging (Bournaud et al. 2005). For instance, ellipticals have been shown to result from the merging of two galaxies of similar masses. However, the inclusion of gas in the simulations now leads to other possible formation scenarii (Bournaud et al. 2007, Ocvirk et al. 2008).

Other classifications of galaxies are somehow inherited from this morphological Hubble classification and are most often dictated by the instrument used to make the observations of the sample. Correlation plots are used to make crude classification in a very few number of categories (often 2 like blue and red, high and low intensity for a given wavelength, more or less metallic, ...). This is most of the time sufficient for some physical modelling, but it cannot describe the huge diversity of galaxies across the Universe and their now recognized complex history in a very objective and synthetic way.

The understanding of galaxy formation and evolution, which we prefer to call galaxy diversification, is a major challenge of contemporary astrophysics. Abandoning the one-parameter classification approach and using all available descriptors means taking a methodological step equivalent to the one biologists took after Adanson and Jussieu in the 18th century. Today the tools do exist, and ordination methods, essentially the one of Principal Component Analysis, are being used more often, mainly to automatically separate stars and quasars from galaxies on large images of the sky (e.g. Cabanac et al. 2002). A few attempts to apply clustering methods have been made recently, still with little success in identifying new classes that convince the astronomical community (e.g. Chattopadhyay \& Chattopadhyay 2006). From our point of view, there are two difficulties here. The first one is that the PCA components are non-physical, hence very difficult to interpret and model in the way astrophysicists are used to. The second one, more important in our point of view, is that evolution, an unavoidable fact, is not at all taken into account. By mixing together objects at different stages of evolution, any physical significance of a classification is undoubtedly lost. This was precisely our motivation for developing astrocladistics in 2001 (Fraix-Burnet et al 2006a, 2006b, 2006c). 


\section{The astrocladistics project}

\subsection{A phylogenetic framework for the galaxies}

As we have seen in the Introduction (Sect. 1), the baryonic matter is immersed in the gravitational field that is shaped by the dominating dark matter. Even though its nature is totally unknown, the dark matter is supposedly affected only by gravitation. This is a relatively simple physics and it enabled the first numerical simulations of the cosmological evolution of the gravitational field in the expanding Universe from the observed primordial fluctuations up to the present time. Since matter attracts matter, tiny overdensities, called halos, progressively grow in mass and size by merging together. This is the hierarchical model of formation of the large structures in the Universe.

The evolution of dark matter halos is generally represented by a "merger tree", a typical one being shown on Fig. 1a (Stewart et al. 2008). Many small halos at the top (redshift of about 7 equivalent to about 13 Gyr ago) of the tree merge while time goes downward to yield large dark matter halos observed in galaxy clusters. These merger trees indeed represent the genealogy of a single halo. A schematic representation these trees is often used, but they tend to suggest that small halos disappear with time. This is not true, and the tree on Fig. 1a clearly shows that some of them "survive". Hence, at any epoch in the Universe, halos of different sizes with different merging histories coexist.

From the astrocladistics point of view, the hierarchical evolution of the dark matter halos is better represented on a phylogenetic tree, where mass (or size) is the only criterion for diversity (Fig. 1b). This tree describes the evolution of the environment in which galaxies form and evolve.

Galaxies are made up of baryonic matter which is sensitive to gravitation, but also to electromagnetic, weak and strong interactions, as well as radiation and thermodynamics. Its physics is thus very complex and there are many processes that can strongly affect its gravitational behaviour. Probably because of the first cosmological numerical simulations that were able to take the sole gravitation into account, galaxies are generally thought of as being at the center of dark matter halos. As a consequence, the hierarchical formation scenario seems to apply naturally to galaxies, and has been very popular up to now (e.g. Baugh 2006, Avila-Reese 2007).

However, the old formation scenario for galaxies, called the monolithic model, gains new consideration thanks to new numerical simulations and observations. This model indeed describes the collapse of a cloud of gas, and includes all the necessary physics which is not included in the pure hierarchical model. Merging of galaxies does exist, but it cannot be of the same nature as for the dark matter since the gravitational energy can be transformed into heat, shocks or radiation. Consequently, the diversification of galaxies cannot follow the same simple hierarchical scenario as that of the dark matter represented on Fig. 1.

Astrocladistics makes it clear that the formation and the evolution of galaxies, governed by a complex physics, occur in an environment whose evolution, distinct and largely independent, is governed by the sole gravitation. Galaxies interact between each other while they move in the gravitational field shaped by the dark matter. To a first approximation, galaxies can be good tracers of the dark matter, but as recent observations show, the two can sometimes be well disconnected.

It is still difficult to tell what the very first objects were. Since the Universe was essentially homogeneous at the recombination epoch, the primordial gas was certainly not very clumpy. With time, with the growth of the tiny initial fluctuations in dark matter halos, gas density began to increase in some places. When these seeds became gravitationally bound, we could call them galaxies. It is probable that the first stars formed very rapidly during this process. These very first galaxies were probably already quite diverse in mass content (gas/stars) but very similar otherwise. They also subsequently merged rapidly, interacted strongly with their neighbours, forming new and still more diverse objects. The expansion of the Universe definitively separated large regions that make what we call superclusters and clusters of galaxies. These can be seen as islands in which the corresponding populations of galaxies have evolved on their own, possibly creating some evolutionary divergence.

However, the physics is the same everywhere (cosmological principle), the environment is purely gravitational and differs from place to place only by its shape in the curved space-time. We thus expect a lot of similarities between different "populations" of galaxies due to convergent and parallel evolutions. Unfortunately, our quest to understanding diversification is somewhat complicated by the finite velocity of light. We have not access to the entire Universe, hence to the entire galaxy diverstiy, that is contemporary to us because when galaxies are a bit far away, we see them as they where when the light was emitted. We are like paleontologists, except that we do not have access to the entire present diversity. Nonetheless recent observations seem to suggest that galaxy evolution has been very gentle in the last $1 \mathrm{Gyr}$ or so. 


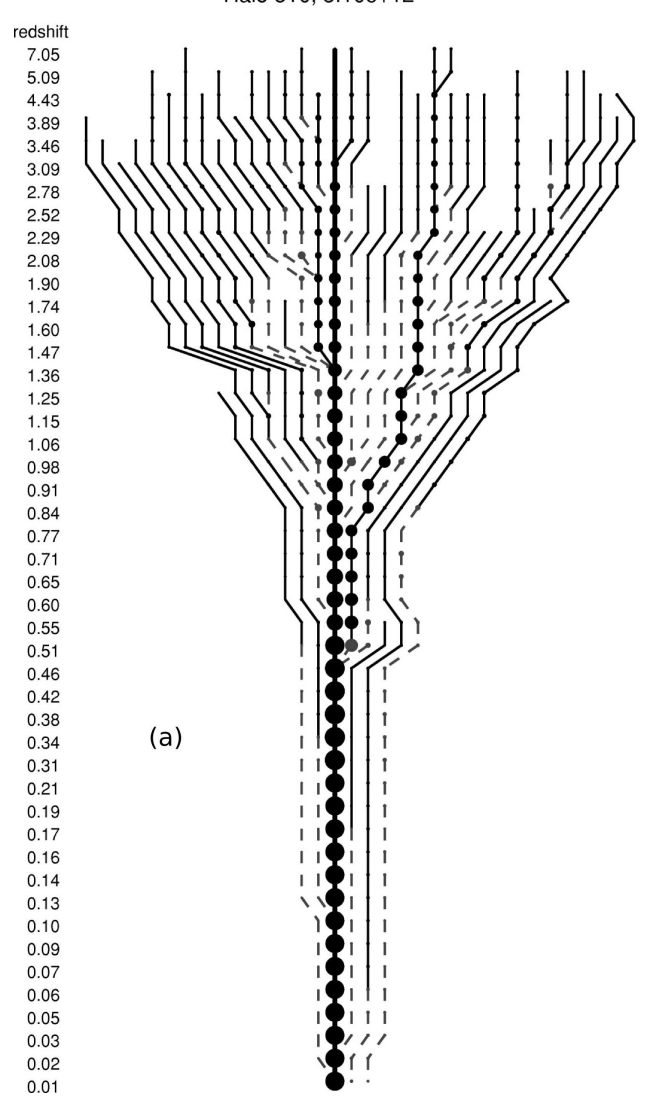

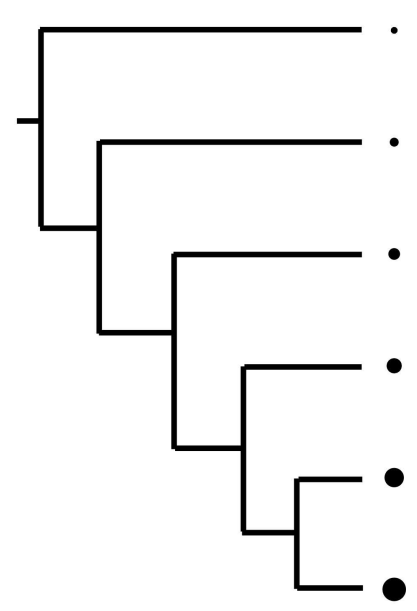

(b)

Figure 1: (a) A "typical" merger tree for an individual dark matter halo with mass $\simeq 10^{12.5}$ solar masses at present time (redshift=0). Time progresses downward, with the redshift printed on the left hand side. The bold, vertical line at the center corresponds to the main progenitor, with filled circles proportional to the radius of each halo. The minimum mass halo shown in this diagram has $\mathrm{m}=10^{9.9}$ solar masses. Solid and dashed lines and circles correspond to isolated field halos, or subhalos, respectively. The dashed lines that do not merge with main progenitor represent surviving subhalos at $\mathrm{z}=0$. Such trees are indeed genealogical trees for individual halos. Taken from Stewart et al 2008 and reproduced by permission of the AAS. (b) The corresponding phylogenetic tree as seen by astrocladistics. There is no time scale in this representation. Mass or size is the sole diversification criterion and increases downward. Each bifurcating node corresponds to a merger event and indicates the first occurrence in the Universe of a halo of a given mass. The chronology thus goes from left to right.

\subsection{Transmission with modification among galaxies}

Galaxy formation is now acknowledged to occur continuously during the Hubble time (Hoopes et al. 2007). In extragalactic astrophysics, evolution and formation are generally not clearly distinguished. Indeed, galaxy evolution is more like galaxy transformation, that leads to the formation of new kinds of objects. Formation often seems to be employed as if big spiral and elliptical galaxies are at the end of evolution. To our point of view, "galaxy formation and evolution" can be more precisely called galaxy diversification, in which evolution means transformation and formation refers to diversity.

There are 5 transformation processes for galaxies: assembling, secular evolution, interaction, accretion/merging, ejection/sweeping. In all these cases, the fundamental material of a galaxy, stars, gas and dust, is transmitted to the new object, somewhat modified. These modifications are mainly kinematical because the gravitational perturbations change the orbits, hence the distribution of the objects. The stars are otherwise unaffected, except that new ones can be formed from the perturbed gas and dust. These latter two constituents can also be modified in density, temperature and composition.

Schematically, galaxy diversification can be depicted like in Fig. 2. At a given time, a galaxy undergoes a transformation event to form a new object made with the same constituents but modified. Between two such 


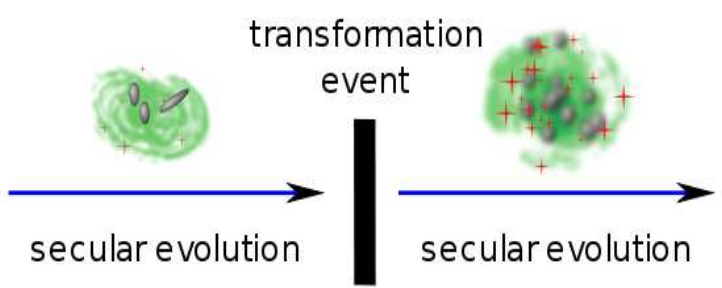

Figure 2: A schematic view depicting the transmission with modification process in galaxy diversification. For any transformation event, gas, dust and stars are transmitted to the new object generally with some modification of their properties. Secular evolution is defined as the evolution of a totally isolated galaxy. It thus occurs all the time, and even if it is not violent in general, it can sometimes lead to a significant transformation.

events, stars get older and essentially cooler (redder). This is part of the secular evolution, which is the evolution of a totally isolated galaxy. Secular evolution is a transformation process driven by internal phenomena (stellar ageing, interaction between the three constituents, density waves, shock waves due to supernovae, instabilities, ...) which is generally slow and non-violent. However, after a certain time, the galaxy can be so different that it can be described as a new object and even belong to a different class of objects. Like for living species, the modifications are gradual and at some level of details, the splitting in different species is somewhat arbitrary. Thus, secular evolution can also be a transformation event participating to the diversification of galaxies.

Figure 2 shows that it is possible to see the transformation of galaxies as a transmission with modification process. It is not exactly a darwinian process in the sense that there is no natural selection (even though big galaxies survive more easily and tend to swallow small ones!). Also, there is no duplication, but each transformation process is a replication mechanism, because the new object has inherited all constituents and most of their properties. Since the transformation events are always random, this is equivalent to a replication with "spontaneous mutations". In addition, the environment, which is gravitational, has a strong influence on the occurrence and properties of all the galaxy transformation processes.

Galaxy diversification is thus characterized by replication, randomness and environment. It follows a branching pattern can be expected. This is why cladistics should be applicable to this problem. However, it might not be so simple since mergers are probably frequent at least near the beginning of the Universe, so that reticulation ("hybridation" or horizontal transfer) could be present. Cladistic analyses of several samples of galaxies can investigate this. As shown in Fraix-Burnet et al. (2006a, 2006b) and confirmed by all the robust cladograms we have obtained so far, reticulation does not seem to be dominant. Yet, this conclusion must be seen as preliminary and more investigation should be done, particularly with very distant objects.

\section{Applying cladistics to galaxies}

Astrophysics is an observational science, not an experimental one. It is not possible to weigh a galaxy or to return it to see what is behind. We simply collect photons, at all wavelengths (from gamma-rays and X-rays to the radio domain, with the ultraviolet, optical, infrared, far-infrared and sub-mm in between), that are emitted by every component at every place in a galaxy. The level of details is limited by the faintness and the small apparent projected size of the objects, particularly those at very high distances from us. Both improve with the diameter of the photon collector (the mirror of the telescope) and with the sensitivity of the detectors. Requirements for the astronomical observations are a strong thrust for technology research and development, and recent progresses in this domain have changed the scale in the amount of data concerning galaxies. But still, each picture element ("pixel", i.e. the smallest detail) is a mixture of the light emission from many stars and gas/dust clouds situated along the line of sight and across a region which size depends on the distance of the galaxy.

Basically, observations result in the recording of spectra. Apart from the structure, given by imagery or relative spatial positioning, spectra contain all the physical and chemical information that is possible to get from remote objects. Systematic surveys of the sky now provide spectra for millions of galaxies. However, in addition to the limitations mentioned above, there are limits coming from the spectral resolution of the detectors and from Doppler effects due to the differential motions of the galaxy components that enlarge and blur absorption and emission lines. As a consequence, physical and chemical conditions at each part of a galaxy are always partial and averaged.

Measuring characteristics from spectra is also not sufficient to derive physical or chemical quantities, some 
combinations must be used like ratios of several emission lines. Moreover, models must most often be used to disentangle the complexity of the emitting region and to translate spectral information into physical quantities. Unfortunately, these models necessarily introduce some subjectivity. Thus, truly intrinsic descriptors of galaxies are not easy to obtain, and this is certainly a specific difficulty of astrocladistics.

Anyhow, descriptors in extragalactic astrophysics are always continuous variables since, apart from spectral information, the other information we can measure is the dimension (size and position of individual components within the galaxy). The only exception is the morphology. It is traditionally based on the Hubble classification represented by the Hubble diagram, most often with the use of a discrete scale distinguishing different kinds of elliptical and spiral galaxies. However, in our astrocladistic project, we have chosen not to use this parameter for several reasons. The first one is that this is a very crude way of discretizing a shape parameter because it is essentially made by eye. The second reason is that this discretization is not homogeneous in the sense that differences between spirals are given the same weight as between ellipticals, while they are not of the same nature (shape and number of the spiral arms which are density waves, not physical structures, for the first ones, and estimation of roundness for the latter ones; de Vaucouleurs 1994). The third reason is that it is not clear at what level this descriptor can be informative regarding evolution. Finally, we know that the Hubble classification is correlated with quite a few global properties of galaxies (kinematics, amount of gas and star formation most notably), so it is certainly redundant with other descriptors, quantitative and objective, coming from the spectrum.

For such an exploratory project like astrocladistics, choices have to be made right at the beginning to focalize the research. Up to now, we have chosen to discretize all observables into 10 or 30 bins, depending on the error bars estimated by the observers and on the distribution of values among the sample. This allows us to use parsimony as the optimisation criterion, which seems to be the simplest strategy to implement for a cladistic analysis. These first choices already enabled us to obtain very positive results. Other paths can now be explored.

Last but not least, we do not have a multivariate classification of galaxies at our disposal. We have to assume that each galaxy represents a "species" that will have to be defined later on. It is consequently difficult to define an outgroup that is necessary to root the tree and help the interpretation of the evolutionary scenario. Most generally, we root the trees with the object that is the less metallic, that is in which the relative abundance of heavy atomic elements is the lowest (heavy elements are only produced in stars, and cannot be primordial). But we are aware that this choice is made on one character only.

\section{The first extragalactic trees}

The first application of astrocladistics was performed on a sample of galaxies issued from a cosmology simulation. This work used 50 objects and 50 characters (observables), corresponding to 5 "lineages" sampled at 10 epochs. In such simulations, each entity, called a galaxy, that appears, is given a number. Only accretion or merging are considered, and a new number is attributed each time such an event occurs. It is then easy to follow the tree of transformations of an initial galaxy into its different descendants. The simulations calculate the radiation from galaxies and transpose it into the same observables as obtained from real telescopes. This work was intended to illustrate the concepts and practical methodology of astrocladistics, and to show how the correct "'genealogy" can be reconstructed (Fraix-Burnet et al. 2006a, 2006b) from usual observables.

More importantly, a small sample of real galaxies (Dwarf Galaxies of the Local Group, 36 objects, 24 characters) have also successfully been analysed, providing the first evolutionary galaxy tree ever established (Fraix-Burnet et al. 2006c). These dwarf galaxies are very numerous throughout the Universe. Most of them are satellites of big galaxies like our own. They supposedly have been the first galaxies at the beginning of the Universe and are thus believed to be the building blocks that merged to make bigger and bigger galaxies. A fully resolved tree obtained on a subset is represented in Fig. 3. Bootstrap and decay values indicated at the nodes show that it is very robust. The groupings are consistent with our current knowledge of these particular objects, with a more refined classification. This tree clearly implies that the nature of the ancestors are of irregular shape. The other type, the spheroidals, are consequently more evolved. This question is debated in the astrophysical community and is naturally addressed by astrocladistics in a multivariate way. It is important to note that the characters used in this analysis are a mixture of observables (like colours, fluxes in different spectral lines and composition ratios) and derived quantities (like masses, maximum rotational velocities, velocity dispersion, star formation rate). Such kind of information is more difficult to obtain with other more distant galaxies.

We subsequently performed a cladistic study of 222 galaxies of the Virgo cluster using 41 characters that were all observables. Because of too many unknown parameters, we were able to build a robust tree for 123 objects only, and used it to construct a supertree. Figure 4 shows the cladogram with 123 galaxies. It took us some time to understand the astrophysical consequence of the regularity of the (largely unbalanced) tree that is also found on 


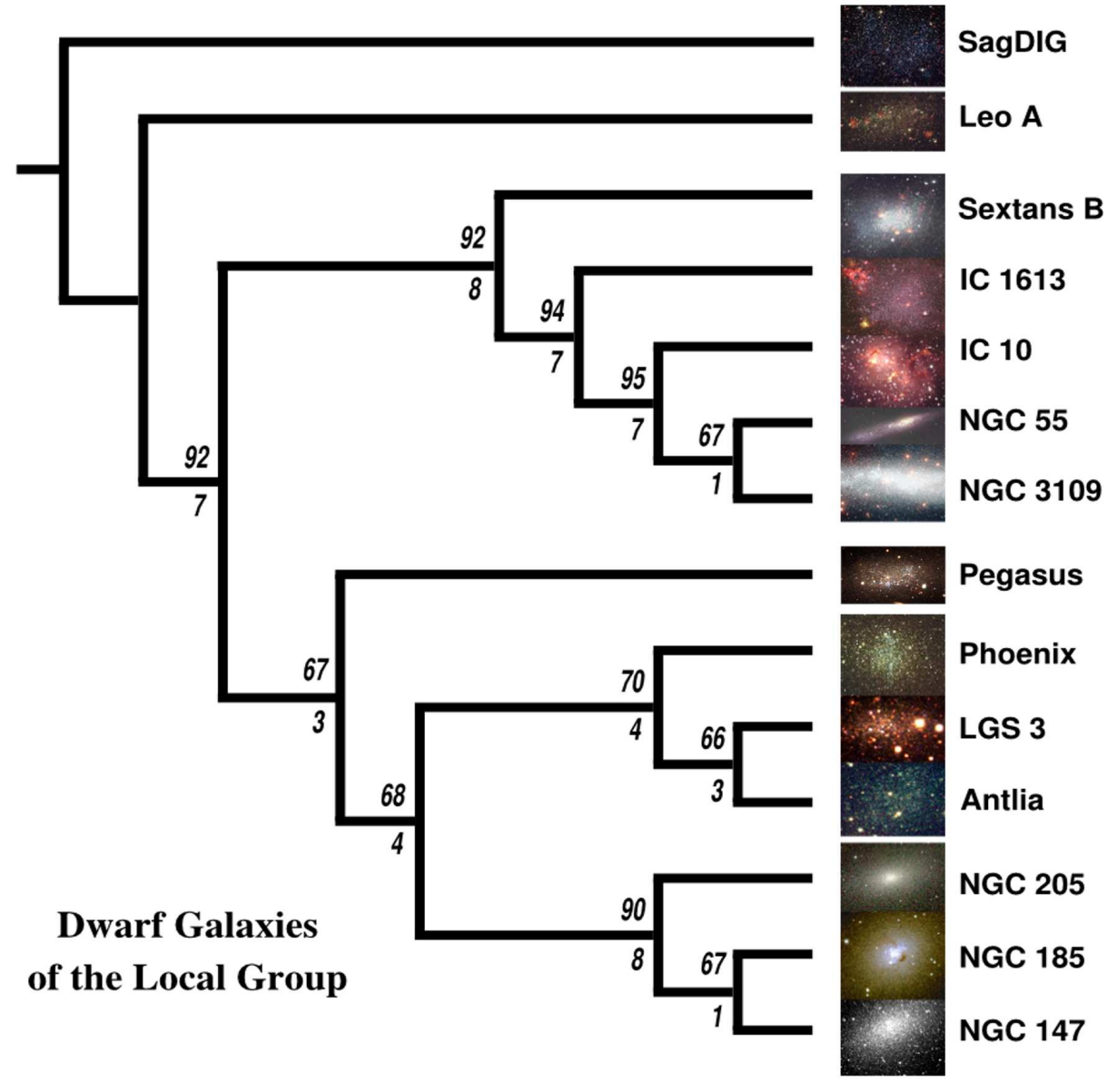

Figure 3: Cladogram of 14 Dwarf galaxies of the Local Group obtained with 24 characters (observables and derived quantities). Bootstrap values (above) and Decay indices (below) are indicated for each node. The outgroup (SagDig) has been chosen because it contains the lowest amount of metallic material, suggesting that it is made up of more primordial material (see Fraix-Burnet et al 2006c for details on this work). Image credits: NGC 55 : David Malin; LGS 3 and Pegasus DIG: Deidre A. Hunter; Antlia: Mike Irwin; NGC 185: David M. Delgado; NGC 147: Walter Nowotny; Sag Dig: Hubble Heritage Team (AURA / STScI), Y. Momany (U. Padua) et al., ESA, NASA; Leo A, Sextans B, IC 1613 and IC 10: Corradi, R.L.M. et al., 2003, ING Newsletter No. 7, p. 11; NGC 3109: NASA/ STScI; Phoenix: Knut Olsen (CTIO) \& Phillip Massey (Lowell Observatory), (NOAO / CTIO / KPNO); NGC 205: Atlas Image [or Atlas Image mosaic] courtesy of 2MASS/UMass/IPAC-Caltech/NASA/NSF.

the supertree. In fact, this is due to the sole stellar evolution which is hidden in nearly all of the observables. It is consequently highly redundant and dominant in this multivariate analysis. The astrophysical outcome is thus slightly disappointing. We obtained similar results on other samples: poor gas galaxies of the field (comprising 227 objects and 37 characters), galaxies from a big survey (500 objects with 60 characters analysed so far).

From the astrocladistic point of view, all these studies shows that our approach is successful in constructing cladograms of galaxies in several different types of samples. Nevertheless, the regular trees found when using solely observables reveal that stellar evolution is universally present and acts as a parallel evolution. It does not bring much information on galaxy diversity, and disturbs the multivariate analyses. We call it "cosmic evolution" because it is universal and does not lead to a sufficient transformation of the galaxy, like secular evolution in general or the other transformation processes. Even in the most violent cases, a given stellar population continues to age unperturbed and its radiation properties are unaffected unless the population itself is changed for instance with the addition of newborn stars. Somehow, we could think the cosmic evolution as a kind of ontogeny for galaxies. We looked for a way to remove it from the observables because it obviously hampers the search for "speciation". We used various synthetic stellar populations, modelling artificial lineages with specific properties. We restricted ourselves to small samples (30 objects). Our idea was to scrutinize each observable in a very well controlled sample. By 

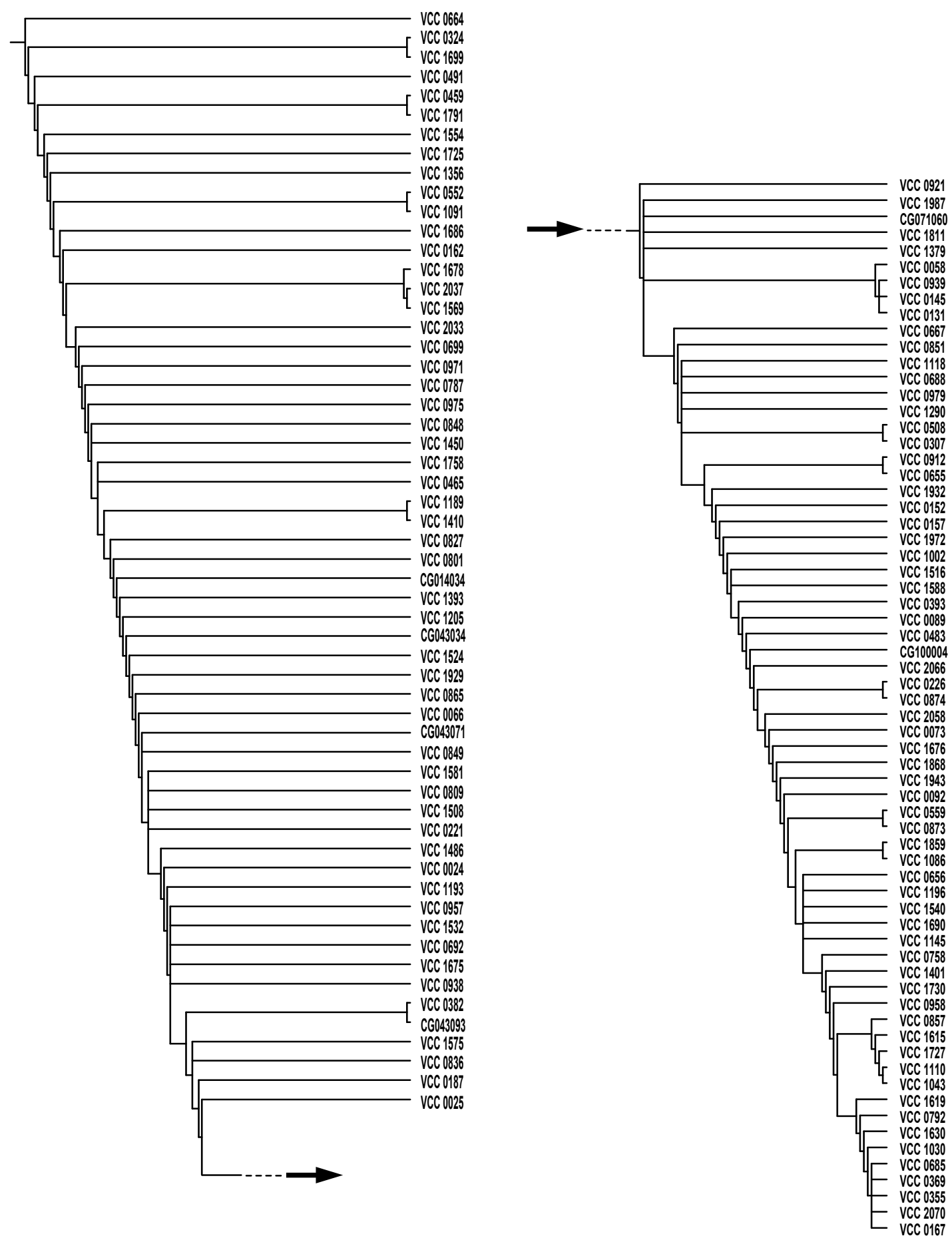

Figure 4: Cladogram for 123 galaxies of the Virgo cluster with 41 observables. Note the regularity of the tree indicating the cosmic evolution (star ageing) which is highly redundant. 
trials and errors, we found some combinations of several observables that make the corrected characters to remain essentially constant between transformation events (Fraix-Burnet et al. in prep). The physical interpretation of these corrected characters is still unclear, but the important result is that they lead to physically consistent clades for these simple synthetic stellar populations. Application to real objects is under progress.

Globular clusters are self-gravitating ensembles of stars without dust or gas. In a sense, they are simple galaxies, somehow like our synthetic stellar populations. Taking advantage of our experience gained so far, we performed a cladistic analysis of 54 globular clusters of our Galaxy by choosing 4 properties. Three of them are intrinsic and comes from their conditions of formation. They are not affected by cosmic evolution. The other one is the colour and measures the age of the stars, it is thus a direct indicator of the cosmic evolution. We gave it a weight half of the other ones, and effectively rank the clusters chronologically within the lineages or groupings that are defined by the other three characters. Our result tree gathers the objects in a new classification that brings a very convincing scenario for the formation of these objects and consequently for some episodes in the building up of our own galaxy. This result is the first spectacular outcome of astrocladistics and will hopefully convince more astronomers to invest in this novel approach (Fraix-Burnet, Davoust \& Charbonnel 2009).

\section{Some open questions}

It is now clear that cladistics can be applied and be useful to the study of galaxy diversification. Many difficulties, conceptual and practical, have been solved, but many remain in the exploration of this large research field. To begin with, it is well possible that probabilistic methods, instead of parsimony, might be better suited for the quantitative nature of the variables and the evolution of galaxies. In this respect, the use of the continuous data without discretization will have to be investigated seriously, even with parsimony (Wiens 2001, MacLeod 2002, Goloboff et al. 2006, Gonzàlez-Rosé et al. 2008).

There are difficulties that seem to be intrinsic to astrophysics. Most notably, we have millions of objects, but a few tens of descriptors. Of course the situation will improve with time, in particular with integral-field spectroscopy (spectra of detailed regions in the galaxy, e.g. Ensellem et al. 2007). Spectra might not be currently employed at their full capacity of description. But it is not clear whether they would lead to the discrimination of hundreds of classes. Perhaps this is an erroneous target, perhaps galaxies cannot be classified with such a refinement. But this is already a matter of using multivariate clustering methods and interpreting their results usefully. We are convinced that improvements can be made here. In any case, cladistics is supposed to identify clades, that are evolutionary groups, whereas the concept of "species" is not defined at all in astrophysics, and we have even not converged toward groupings based on multivariate analyses.

This is probably more than a conceptual question because clustering with continuous data and large intraspecific variance is a very complicated problem in itself. Sophisticated statistical tools must be used, but the question of characterizing the groups in this context is not very clear to us at this time. This probably requires a different culture that is not yet present in astrophysics. Incidentally, this also points to the relevance of discretization we have used so far particularly when measurement uncertainties are sometimes quite large. Our results definitively show that we are not wrong, but it might be possible to do better.

The possible predominance of reticulation is a concern for cladistics in general, and we foresee to investigate this question very soon. In some ways, galaxy diversification has similarities with bacteria diversification for which reticulation methods have been considerably developed recently. We intend to take advantage of these advances. It would already be interesting to detect some reticulation in the samples we have studied so far. The equivalent of hybridation or horizontal transfer certainly comes through the merging of two galaxies. Since the Universe is in expansion, object density was higher at earlier times, and interactions and merging were more common. As a consequence, reticulation could be a problem for distant galaxies, that is when the Universe was young.

A very important step for astrocladistics is our assessment of the influence of the cosmic evolution and our ability to correct for it or avoid it in the character selection. But there are probably other kinds of parallel or convergent evolutions, and reversals as well. Hence, we are now concentrating our efforts on finding the most pertinent descriptors. For this we must detail all the possible quantities we can get from the observations, to see how they behave during the different transformation processes and whether they can be reliable tracers of past events from a cladistic point of view. Biologists pretty well know that this is a crucial work, apparently without an end. 


\section{Conclusion}

The astrocladistic project has now reached a solid base as far as concepts and tools are concerned. First results have shown the usefulness of this approach, and unavoidable difficulties are being solved one after one. This triggers the hope of being able to map galaxy evolution from the very first objects of the Universe, some 14 Gyr ago, but there is still a long way to go. Astrocladistics opens a new way to analyse galaxy evolution and a path towards a new systematics of galaxies.

Extragalactic astrophysics is currently undergoing a scaling revolution. The number of objects and the amount of data it has to cope with already impose new statistical tools to be used, and this will certainly get worse with still bigger telescopes and more sensitive detectors. Less than a century after their discoveries, galaxies come by millions in our databases. This is a cultural revolution, in particular because the complexity of these objects and their evolution become more obvious.

Astrocladistics is fascinating in combining a multivariate statistical philosophy with evolutionary concepts. It is fascinating as an interdisciplinary research field. But it is also quite a difficult approach, not intuitive at all, and does not fit well in the usual methods of physicists. This is a huge research field, very exploratory, with many possible paths to follow. We still lack spectacular astrophysical outcomes, but most astrophysicists are very enthusiastic, because they agree that there is an obvious need for multivariate and evolutionary methods to study galaxy diversification.

\section{References}

Avila-Reese V. (2007) Understanding Galaxy Formation and Evolution. Ap\&SS Proceedings, Solar, stellar and galactic connections between particle physics and astrophysics, Carramiñana A, Guzmán F S, Matos T (eds), Springer (2007), 115-165. http://arxiv.org/abs/astro-ph/0605212

Baugh C.M. (2006) A primer on hierarchical galaxy formation: the semi-analytical approach. Reports on Progress in Physics, Volume 69, Issue 12, 3101-3156. http://arxiv.org/abs/astro-ph/0610031

Bournaud F., Jog C.J., Combes F. (2005) Galaxy mergers with various mass ratios: Properties of remnants. Astronomy and Astrophysics 437, 69-85. http://arxiv.org/abs/astro-ph/0503189

Bournaud F., Jog C.J., Combes F. (2007) Multiple minor mergers: formation of elliptical galaxies and constraints for the growth of spiral disks. Astronomy and Astrophysics 476, 1179-1190. http://arxiv .org/abs/0709. 3439

Cabanac RA, de Lapparent V, Hickson P (2002) Classification and redshift estimation by principal component analysis. Astronomy \& Astrophysics 389, 1090-1116. http://arxiv.org/abs/astro-ph/0206062

Cecil G., Rose J.A. (2007) Constraints on Galaxy Structure and Evolution from the Light of Nearby Systems. Rep. Prog. Phys. 70, 1177-1258. http://arxiv.org/abs/0706.1332v2

Chattopadhyay T., Chattopadhyay A.K. (2006) Objective classification of spiral galaxies having extended rotation curves beyond the optical radius. The Astronomical Journal 131, 2452-2468.

de Vaucouleurs G. (1994) Global Physical Parameters of Galaxies. In: Quantifying Galaxy Morphology at High Redshift. Space Telescope Science Institute (Baltimore, USA), April 27-29 1994.

http://www.stsci.edu/institute/conference/galaxy-morphology/program2.html.

Emsellem E., Cappellari M., Krajnović D., van de Ven G., Bacon R., Bureau M., Davies R.L., de Zeeuw P.T., Falcón-Barroso J., Kuntschner H., McDermid R., Peletier R.F., Sarzi M. (2007) The SAURON project - IX. A kinematic classification for early-type galaxies. Mon. Not. R. Astron. Soc. 379, 401-417. http://arxiv.org/abs/astro-ph/0703531v3

Fraix-Burnet D, Choler P, Douzery E, Verhamme A (2006a) Astrocladistics: a phylogenetic analysis of galaxy evolution. I. Character evolutions and galaxy histories. Journal of Classification 23, 31-56. http://arxiv.org/abs/astro-ph/0602581

Fraix-Burnet D, Douzery E, Choler P, Verhamme A (2006b) Astrocladistics: a phylogenetic analysis of galaxy evolution. II. Formation and diversification of galaxies. Journal of Classification 23, 57-78.

http://arxiv.org/abs/astro-ph/0602580 
Fraix-Burnet D, Choler P, Douzery E (2006c) Towards a Phylogenetic Analysis of Galaxy Evolution : a Case Study with the Dwarf Galaxies of the Local Group, Astronomy \& Astrophysics 455, 845-851.

http://arxiv.org/abs/astro-ph/0605221

Fraix-Burnet, D., Davoust, E., Charbonnel, C. (2009) The environment of formation as a second parameter for globular cluster classification. Monthly Notices of the Royal Astronomical Society, in press.

http://fr.arxiv.org/abs/0906.3458

Goloboff P.A., Mattoni C.I., Quinteros A.S. (2006) Continuous characters analyzed as such. Cladistics 22, $589-601$.

Gonzàlez-José R., Escapa I., Neves W.A., Cúneo R., Pucciarelli H.M. (2008) Cladistic analysis of continuous modularized traits provides phylogenetic signals in Homo evolution. Nature 453, 775-779.

Hernandez X, Cervantes-Sodi B (2006) A dimensional study of disk galaxies. 11th Latin-AmericanRegional IAU Meeting 2005, RevMexAA (Serie de Conferencias), 26, 97-100. http://arxiv.org/abs/astro-ph/060225

Hinshaw G., Weiland J.L., Hill R.S., Odegard N., Larson D., Bennett C.L., Dunkley J., Gold B., Greason M.R., Jarosik N., Komatsu E., Nolta M.R., Page L., Spergel D.N., Wollack E., Halpern M., Kogut A., Limon M., Meyer S.S., Tucker G.S., Wright E.L. (2009) Five-Year Wilkinson Microwave Anisotropy Probe (WMAP) Observations: Data Processing, Sky Maps, and Basic Results. Astrophysical Journal Supplements180:225-245. http://arxiv.org/abs/0803.0732

Hoopes C.G., Heckman T.M., Salim S. et al. (2007) The Diverse Properties of the Most Ultraviolet-Luminous Galaxies Discovered by GALEX. Astroph. J. Suppl. Series 173, 441-456. http://arxiv.org/abs/astro-ph/0609415

Kashlinsky A., Arendt R.G., Mather J., Moseley S.H. (2005) Tracing the first stars with fluctuations of the cosmic infrared background. Nature 438, 45-50. doi:10.1038/nature04143

MacLeod N. (2002). Phylogenetic signals in morphometric data, pp. 100-138. In: MacLeod N., and Forey, P.L. (eds.), Morphology, Shape, and Phylogeny. Taylor \& Francis, London.

Ocvirk P., Pichon C., Teyssier R. (2008) Bimodal gas accretion in the Horizon-MareNostrum galaxy formation simulation. Mon. Not. R. Astron. Soc. 390, 1326-1338.

Stewart K.R., Bullock J.S., Wechsler R.H., Maller A.H., Zentner A.R. (2008) Merger Histories of Galaxy Halos and Implications for Disk Survival. The Astrophysical Journal 683, 597-610. http://arxiv.org/abs/0711.5027

van den Bergh, S. (1998) Galaxy Morphology and Classification. Cambridge University Press

Wiens J.J. (2001) Character Analysis in Morphological Phylogenetics: Problems and Solutions. Syst. Biol. 50, 689-699. 